\title{
SYNTHESIS OF PARTITION-CODEC ARCHITECTURE FOR LOW POWER AND SMALL AREA CIRCUIT DESIGN
}

\author{
Shanq-Jang Ruan, Jen-Chiun Lin, Po-Hung Chen, Kun-Lin Tsai, Feipei Lai \\ Dept. of Electrical Engineering \& \\ Dept. of Computer Science and Information Engineering \\ National Taiwan University Taipei 106, Taiwan. \\ flai@cc.ee.ntu.edu.tw
}

\begin{abstract}
Partitioning circuit for low power design in logic level has been proposed as a very effective technique. However, the increased area of latches for duplicated input of multiple partitions always offsets the advantage. In this paper we propose a novel Partition-Codec Architecture to achieve low power and small area. The approach is based on evenly partition the output vectors by the corresponding input variables and re-assigning the output vectors of each partition to minimize the number of input vectors and Hamming distance of each partition, and one of the active decoders returns the value to its original output. Given a combinational circuit described by PLA, we develop a global-encoding algorithm, which consists of partition and re-assigning routines to synthesize the Parition-Codec architecture to achieve low power and small area. Experimental results show that up to $69.5 \%$ power reduction, as well as $60.9 \%$ area decreased and average $35.7 \%$ power saving with $58.4 \%$ area reduction are achevable.
\end{abstract}

\section{INTRODUCTION}

In this paper, we are concerned with optimizing logic level combinational circuits for low power. At this level, there are two major low power techniques: gated-clock and precomputation. Luca and Giovanni proposed gated-clock to build low power FSMs [1]. The technique stops the useless circuit glitches in the idle states of FSMs. In [2], [3], an FSM is decomposed into a number of coupled submachines so that some state transitions of high probability will be confined to the smaller submachines most of the time. Alidina, et al. first proposed the precomputation-base scheme, which selectively disables the partial input of a sequential logic circuit to obtain power saving [4]. On the other hand. another variant precomputation scheme is circuit partition. Choi and Hwang partition a combinational circuit into multiple subcircuits through recursive application of the Shannon expansion with respect to the selected input variables
[5]. The advantage of this architecture is that it simplifies the precomputation logic as a "decoder". Nevertheless, the algorithm to select the best input is quite complex and the duplicated input latches is a significant overhead. Ruan, et al. showed that the partitioning circuits more than two-way often lose advantage because of the overhead of duplicated input latches and output multiplexors in area and power [6]. We proposed a bipartition-codec architecture to reduce the power consumption by re-encoding the output of the small subset of the output vectors, however, additional circuit always increases the area and offsets the power saving [7].

In this paper, we propose a partition-codec architecture for optimizing the power and area of combinational circuits. The technique is based on evenly partitioning the output vectors by its input variables, where each of them is significantly smaller than the original. Since the output vector number of each partition may be reduced, we develop a global-encoding approach for partitioning and re-assigning the output vectors to minimize the number of input pins of each partition.

\section{PARTITION-CODEC ARCHITECTURE}

We propose a partition-codec architecure as illustrated in Fig. 1, which retains the advantage of the Shannon expansion scheme, however, the new architecture shortens the length of each input by applying an Encoder.

The principle of partition-codec architecture can be described as follows: in Fig. 1, the subset of the input pins $I_{s}$ are used as the selection logic for ensuring only one of the decoders is enabled at the same time. The function of the encoder is to encode the output with minimal number of bits and achieve less switching activity. Finally $I_{s}$ drives the select lines of a multiplexor to choose the correct output among the decoders.

As a result, the algorithm to select the input variables determines the number of the latches needed for this architecture. On the other hand, the encoding algorithm for en- 


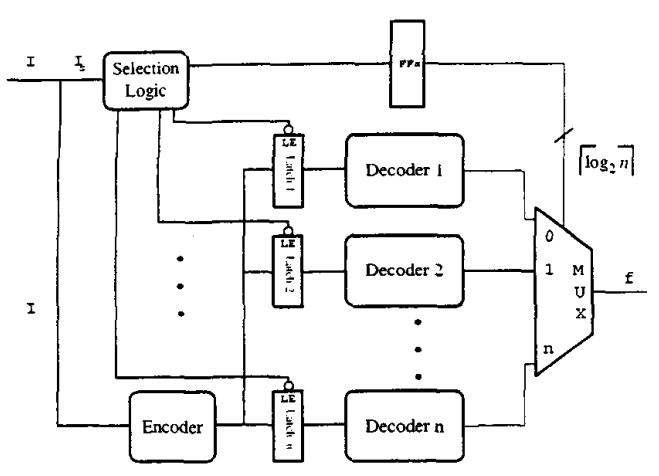

Figure 1: Partition-codec architecture.

coder will affect the circuit complexity and the switching activity of the latch. Hence, the power saving effect of our architecture heavily depends on these two algorithms. In the next section. we will address the problems.

\section{GLOBAL-ENCODING ALGORITHM}

\subsection{Problem Formulation}

From [8], the area complexity strongly depends on the number of input, in which, the average area complexity of an $n$-input Boolean function varies exponentially with $n$. Thus if we can minimize the number of input of each partition (named "decoder" in Fig. 1), we can efficiently reduce the power dissipation. On the other hand, the encoder is a main source of power dissipation because it will be activated every cycle. It can be optimized with the encoding output algorithm by reducing the entropy of the output and area complexity, however, the number of output of encoder is determined by the maximal number of different output of partitions. Therefore, we need to consider partition and encoding at the same time for finding a global solution. According to the above discussion, the global encoding problem is composed of two phases:

1. Partition phase: Based on the Shannon expansion. we select the input variables which minimize the different output number of each partition.

2. Encoding phase: Since we partition circuit according to the criterion of evenly distributing distinct output to each partition, an optimal number will be obtained. Our goals of encoding are: first minimize the switching activity of the output/input pin of encoder/decoder, in which the switching activity of latches is also reduced. Second, minimize the area of encoder.

\subsection{Partition Algorithm}

Consider a circuit with $n$ input pins, if we partition circuit into $2^{k}$ subcircuits by using the Shannon expansion, there will be $\left(\begin{array}{l}n \\ k\end{array}\right)$ different combinations. It is very inefficient to try all these combinations for finding an optimal solution. Thus we develop a heuristic approach to find the minimal number of output for each partition. The basic operation is selecting an input variable which yields the even distribution with minimal number of different output. At the beginning, a new input set is given without selecting variable for partitioning. A $2^{k}$ partition will be achieved by applying the operation $k$ times. The following heuristic partition algorithm illustrates the procedure.

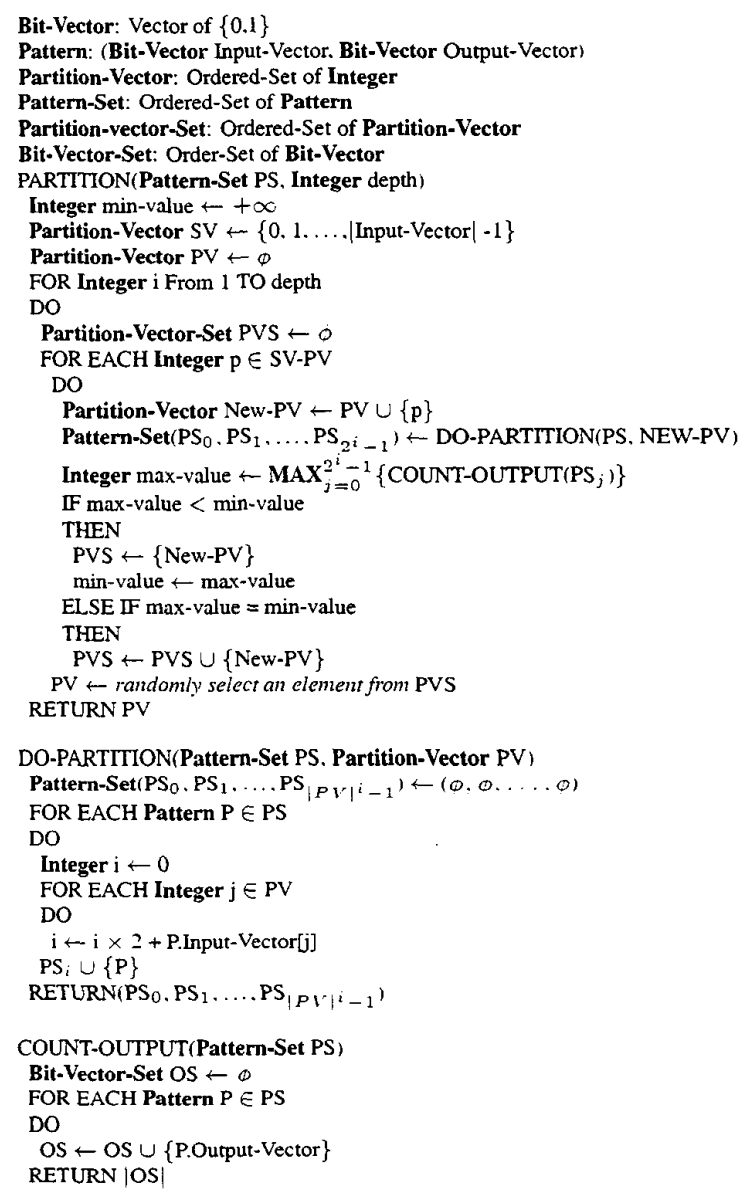

The algorithm, PARTITION, partitions a circuit into 2 depth partitions according to the depth bits of its input. The outer loop adds one more input variable to the partition vector such that the number of different output of partitions is minimal, until we reached depth bits. In the algorithm, the $D O$ PARTITION routine partitions the circuit into $2^{i}$ subcircuits with respect to newly added input variable, at last we re- 
turn the partition result. The max function returns the maximal number of different output among the partitions which counted by COUNT-OUTPUT routine. This value determines the partition performance with the newly added input pin. The IF condition of PARTITION routine compares the partition performance with the previous ones. The smaller, the better. If the chosen variable results in minimal value, it will replace the old chosen variable in this level. Note that if there exist more than one bit of the same performance, we randomly select one in our algorithm. Hence the inner loop finally chooses an input variable which results in the evenly distributed output. Finally, the selected input bits form the partition vector $P V$ which determines the partition result of a given circuit. In fact, our algorithm is a greedy method which yields an optimal solution for evenly partitioning circuit in terms of output.

\subsection{Encoding Algorithm}

The purpose of encoding is to re-assign output for each partition generated by partition algorithm with low power and small area consideration. Although there are a lot of researches on optimal encoding, the problem we encounter is much complexer than that. For completing the binary code assignment, two phases are needed for our encoding consideration: first we have to assign a symbol to the same output of each partition. Second, encoding each symbol to a binary code. The following algorithm illustrates the strategy.

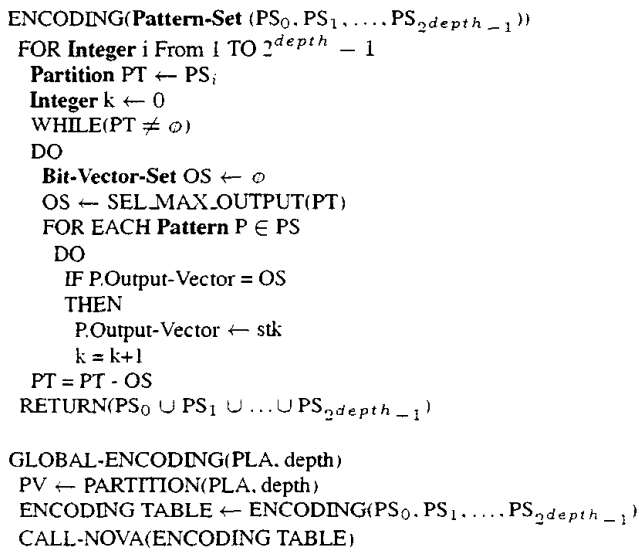

Note that in the ENCODING routine, the SEL_MAX_OUTP $\mathbf{U T}(\mathbf{P T})$ function will return an output vector which is the most frequent occurrence of the pattern PT. The GLOBAL -ENCODING function is the main function of our algorithm. First of all. we call PARTITION routine to partition a PLA table into $2^{\text {depth }}$ sub-PLAs, Second we encode the output of each sub-PLA by the ENCODING routine. Note that this routine returns an encoding table with re-assigning distinct output as a symbol. Finally, we encode the symbols by
NOVA, which is used to obtain an optimal area of encoder [9].

\section{EXPERIMENTAL RESULTS}

The global-encoding algorithm has been implemented in the $\mathrm{C}++$ language and executed on an Ultra Sparc 2 station. In order to verify the results, we use SIS to synthesize and estimate power of each partition and additional control logic by using the command map $-s$ to map a design to the cell library MCNC.genlib and MCNC_latch.genlib[10]. A clock frequency of $20 \mathrm{MHz}$ and $5 \mathrm{v}$ supply voltage were assumed. All power estimates are in micro-Watts and areas are the relative cost (which defined by MCNC.genlib and MCNC_latch.genlib of each cell in SIS). We assumed uniform input probability distribution so that the activation probability of each partition can be regarded as $\frac{1}{n}$, where $n$ is the number of partition. Thus we can calculate total power by summing up the power dissipation of each component in terms of its probability as the following equation:

$$
\begin{aligned}
P_{\text {total }}= & P(\text { Encoder }+ \text { Selection logic }+ \text { Multiplexor })+ \\
& \sum_{i=1}^{n} \frac{1}{n} P\left(\text { Latch }_{i}+\text { Decoder }_{i}\right)
\end{aligned}
$$

where $n$ is number of partitions. Since the function of the selection logic can be regarded as a decoder, which power dissipation is proportional to the number of partitions. The power dissipation of encoder relates to the encoding of output. Moreover, the encoding of encoder output also has significantly impact on the switching activity of latches.

We verify our global-encoding algorithm on several MC $\mathrm{NC}$ benchmarks in two, four and eight-way partitions for comparison. Table 1 presents the experimental results for our architecture. In the table, the number of input and output are labeled as "\#in' and "\#out", power dissipation and area of original circuits are labeled as 'Power" and "Area" in original column. In partition-codec column, the number of partition is labeled as ' $\# P$ ', the power dissipation and area of multiplexor and encoder are labeled as ' $M u x P^{\prime}$ ', 'Mux $A$ ', ' $E P$ ' and ' $E A$ ', respectively. ' $T P$ ' and ' $T A$ ' are the total power dissipation and area. Finally the percentage reduction in power (Power\%) and area (Area\%) are shown. The power and area reductions are computed as $\frac{100 \text { (Original-patition) }}{\text { original }}$. Thus, the negative values of the columns mean the power/area reduction in partition-codec architecture. The power and area of the original circuits and partition circuits include latches at the input ends and control logic (latches, flip-flops, decoders and multiplexor), respectively.

As we can see from the table, a significant power saving can be obtained, up to $69.5 \%$, as well as the $60.9 \%$ decreased area and The average power and area reduction are $35.7 \%$ and $58.4 \%$ in two-way parition-codec architecture, respectively. It is obvious that the extra selection logic and multiplexors always offset the power gain from our architecture. 


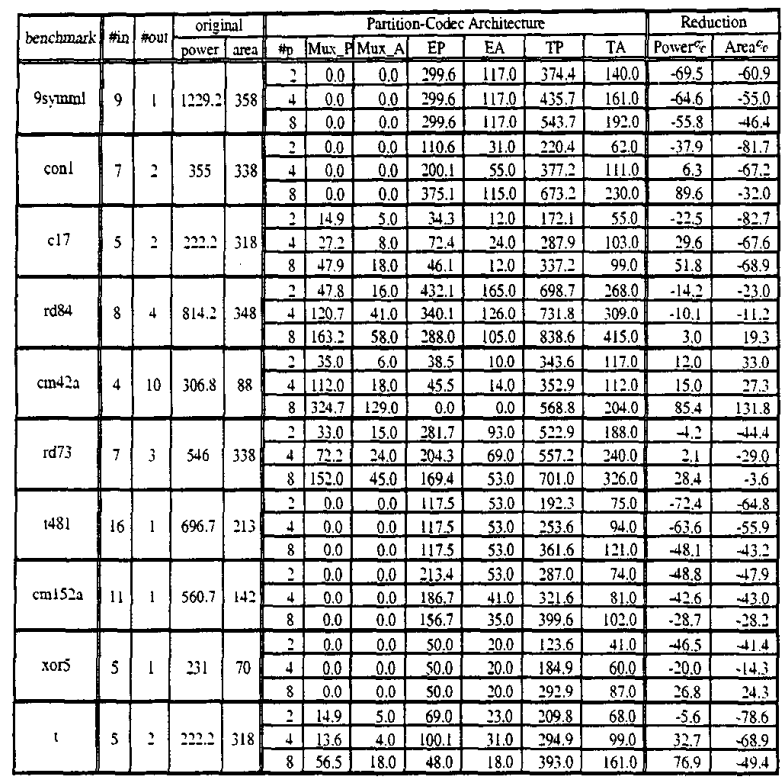

Table 1: Experimental results

We want to point out that our approach is not restricted to large circuits, for example, we can obtain power and area reduction for $\mathbf{c m 1 5 2 a}$ and this benchmark is quite small in area. It should be noted that the power dissipation of encoder is not increased by the number of partitions. The power dissipation of encoder is determined by the circuit property. In fact, if the the number of output pin of encoder is much less than the input number, the encoder dissipated less power. However, the power dissipation of multiplexors is always proportional to the number of partitions. It takes a large portion of total power dissipation and offsets the power saving. Therefore, as proposed in [5], if we replace the output multiplexor by a transmission gate in a wired-OR form, our method can produce better results in power and area consumption.

\section{CONCLUSION}

We have presented a partition-codec architecture in logic level circuit design for low power and small area. For the architecture, we developed a global-encoding algorithm targeting at low power and small area. The results show that power savings of up to $69.5 \%$, as well as $60.9 \%$ in area are possible in some cases and average $35.7 \%$ power and $58.4 \%$ area reduction. Unlike previous study of circuit partition, we want to emphasize that our approach can obtain not only power saving but also area decreased. This is due to the fact that our architecture and global-encoding algorithm can ef- fectively reduce the length of input latches and power/area of each partition (encoder and decoders).

\section{REFERENCES}

[1] L. Benini and G. D. Micheli, "Automatic Synthesis of Low-Power Gated Clock Finite-State Machine," IEEE Trans. Computer-Aided Design, vol. 15, pp. 630-643, Sep. 1996.

[2] J. C. Monterio and A. L. Oliveira, "Finite State Machine Decompostion For Low Power," in Proceeding Design Automation Conf., pp. 758-763, 1998.

[3] S.-H. Chow, Y.-C. Ho, T. Hwang, and C. L. Liu, "Low Power Realization of Finite State Machines- A Decompostition Approach." ACM Tran. Design Automation of Electronc Systems, vol. 1. pp. 315-340, Jul. 1996.

[4] M. Alidina, J. Monterio, S. Devadas, S. Devadas, A. Ghosh, and M. Papaefthymiou, "PrecomputationBased Sequential Logic Optimization for Low Power," IEEE Trans. VLSI Syst., vol. 2, pp. 426-436, Dec. 1994.

[5] I.-S. Choi and S.-Y. Hwang. "Circuit Partition Algorithm for Low-Power Design Under Area Constraint Using Simulated Annealing," IEE Proc. Circuit Devices Syst., vol. 146, pp. 8-15, Feb. 1999.

[6] S.-J. Ruan, J.-C. Lin, P.-H. Chen, F. Lai, K.-L. Tsai, and C.-W. Yu, "An Effective Output-Oriented Algorithm for Low Power Multipartition Architecture," in IEEE Int'l. Conf. on Electronics, Circuits and Systems (accepted), 2000.

[7] S.-J. Ruan, R.-J. Shang, F. Lai, S.-J. Chen, and X.J. Huang, "A Bipartition-Codec Architecture to Reduce Power in Pipelined Circuits," in Proceedings of IEEE/ACM Int'l. Conf. on Computer Aided Design, pp. 84-89, 1999.

[8] K.-T. Cheng and V. D. Agrawal, "An Entropy Measure for the Complexity of Multi-output Boolean Functions," in proceedings of 27th ACM/IEEE Design Automation Conference, pp. 302-305, 1990.

[9] T. Villa and A. Sangiovanni-Vincentelli, "NOVA: State Assignment of Finite State Machines for Optimal Two-Level Logic Implementation," IEEE Trans. Computer-Aided Design, vol. 9, pp. 905-924, SEP 1990.

[10] E. M. Sentovich. "SIS: A System for Sequential Circuit Synthesis," tech. rep., Department of EECS, University of California, Berkeley, CA94720, 1992. 